\title{
PIANOESCALAFORTE: UNA HERRAMIENTA DIGITAL PARA EL APRENDIZAJE DE ESCALAS EN EL PIANO $^{1}$
}

\section{PIANOESCALAFORTE: A DIGITAL TOOL FOR LEARNING SCALES ON THE PIANO}

\author{
Michel André Cara Jara $\left(^{*}\right)$ \\ Instituto de Música \\ Facultad de Filosofía y Educación \\ Departamento de Pedagogía \\ Pontificia Universidad Católica de Valparaiso \\ Chile \\ Ismael Figueroa Palet \\ Universidad de Valparaíso \\ Chile \\ Gonzalo Zeballos \\ Roberto Hormazábal \\ Pontificia Universidad Católica de Valparaiso \\ Chile
}

\section{Resumen}

\begin{abstract}
El presente estudio evalúa la eficacia de la plataforma digital "PianoEscalaForte" (PianoEF) como dispositivo de microaprendizaje de evaluación de escalas en el piano, en el contexto de la clase de piano grupal. Participan en este, 14 estudiantes de primer año de piano del Instituto de Música de la Pontificia Universidad Católica de Valparaíso. Los músicos tocan una escala musical por la primera vez (pre-test) y luego son expuestos a las distintas modalidades de retroalimentación de la plataforma (visual, auditiva y verbal) mientras perfeccionan la ejecución de la escala hasta realizar una versión final (post-test). Los datos de la performance generados vía formato MIDI y el análisis de los cuestionarios retrospectivos aplicados, indican que PianoEF contribuye al desarrollo de la clase de piano grupal, facilita la retroalimentación y ayuda a los pianistas a mejorar la uniformidad de la ejecución musical en términos de intensidad y de articulación (i.e., legato).
\end{abstract}

Palabras clave: Evaluación de escalas; retroalimentación multimodal; plataforma digital de apoyo al microaprendizaje; piano; MIDI.

\section{Abstract}

This study evaluates the effectiveness of the digital platform "PianoEscalaForte" (PianoEF), as a microlearning device for assessing musical scales on the piano, in the context of group piano class. Fourteen first-year piano students from the Music Institute of the Pontificia Universidad Católica de Valparaíso participate in the study. The musicians play a musical scale for the first time (pre-test) and are then exposed to the different feedback modalities of the platform (visual, auditory and verbal) while improving the performance of the scale until making a final version (post-test). The performance data generated via MIDI format and the analysis of the retrospective questionnaires applied, indicate that PianoEF contributes to the development of the group piano class, facilitates feedback and helps students to improve the uniformity of music performance in terms of intensity and articulation (i.e., legato).

Keywords: Musical scales assessment; multimodal feedback; digital platform to support microlearning; piano; MIDI.

\footnotetext{
${ }^{1}$ Los autores agradecen el apoyo de la Vicerrectoría de Investigación y Estudio Avanzados de la PUCV y a los académicos del Instituto de Música participantes en el proyecto: María Angélica Beláustegui, Patricia Escobar, María Angélica Rueda, Manuel Montero y Samuel Quezada.

RECIBIDO: 27.10.2019

ACEPTADO: 20.12.2019

DOI: 10.4151/07189729-Vol.59-Iss.1-Art.1018 


\section{Introducción}

La evaluación objetiva del nivel técnico de un pianista -por ejemplo, a través de la ejecución de escalas- es un tema controvertido dada la diversidad de enfoques, "escuelas" y pianistas. Como resultado, la evaluación pianística requiere una atención particular, lo que implica la cuantificación de ciertas características de la interpretación, tales como: control, velocidad, legato y simultaneidad. En comparación con otras disciplinas, la evaluación musical tiene algunas características específicas que se realizan considerando su naturaleza temporal, sus representaciones culturales y las preferencias estéticas del evaluador. Minder (1996) sostiene que no se puede hacer una evaluación válida, sobre criterios indefinidos, o con referencia a propósitos mal definidos. Por tanto, el problema de la validez de la evaluación en el dominio musical se podría solucionar definiendo criterios y parámetros objetivos que faciliten el diálogo entre la comunidad educativa. En efecto, parte de la dimensión social del aprendizaje consiste en la construcción de sentido, a partir de un proceso de transformación que es regulado, desde un punto de vista pedagógico, por la evaluación (Cara \& Aranda, 2016). Por lo demás, el aprendizaje del piano es un proceso continuo, donde los aspectos técnicos están directamente relacionados con el repertorio y la maduración de las obras. En esto, el trabajo diario juega un papel importante y presupone que se establezcan objetivos a corto y largo plazo. Según Ciccolini y Lafaye (1998), si la técnica no está respaldada por un entendimiento, no es nada, entonces no es posible progresar. Por tanto, otra forma de entender esta noción de progresión es considerar que existe una comprensión intelectual inmediata, que coexiste con la necesidad de un ajuste entre el rendimiento ideal y el rendimiento obtenido. Para fortalecer la comprensión de dichos procesos técnicos podría ser pertinente, desde un punto de vista pedagógico, recurrir a actividades interrelacionadas y de corta duración (5 a 15 minutos) que faciliten el aprendizaje de microcontenidos, lo que se conoce como microaprendizaje (Palazón, 2015).

En el presente trabajo se describe el diseño y evaluación de un primer prototipo de PianoEscalaForte (PianoEF), una aplicación de escritorio para sistema operativo Windows, que permite evaluar la ejecución de escalas en el piano, particularmente las características cuantitativas de dicha evaluación. En primer lugar, revisamos el estado del arte en cuando a los modelos de evaluación de escalas y los elementos técnicos utilizados, luego presentamos el primer prototipo de PianoEF y los análisis realizados. Para finalizar, se discute sobre alcances, limitaciones y perspectivas de trabajo futuro. Dada la estrecha relación entre el control motor fino, el desarrollo de competencias auditivas, la expresividad y el uso de dinámicas en la música (Riley, Coons \& Marcarian, 2005), nos interesa evaluar dichos ámbitos a través del uso de la retroalimentación multimodal, ya que existe evidencia que este tipo de retroalimentación podría aportar mayor efectividad en el proceso de aprendizaje (De Jong, 2010; Nijs \& Leman, 2014). 


\subsection{Escalas musicales}

Una escala musical puede considerarse como una secuencia arbitraria de notas. La interpretación de escalas puede hacerse de diversas formas: con una o ambas manos, en sentido paralelo ambas manos en la misma dirección-, o bien en sentido contrario -una mano asciende mientras la otra desciende-. Es posible interpretar las escalas con distintas articulaciones, por ejemplo: (1) staccato: se acorta la duración de cada nota; (2) legato o ligado de expresión: las notas se deben tocar de manera ligada o sin separación entre ellas; (3) crescendo: la intensidad va aumentando progresivamente a medida que se ejecuta una frase musical, y (4) diminuendo: la intensidad va decreciendo progresivamente a medida que se ejecuta una frase musical.

Existe evidencia de que la realización de ejercicios preparatorios o técnicos, tales como la ejecución de escalas en distintas tonalidades, es una tarea altamente demandante (Vaquero et al., 2016), y que además resulta ser predictiva del nivel de experticia de los pianistas (Jabusch, Alpers, Kopiez, Vault \& Altenmüller, 2009; Jabusch, Vauth \& Altenmüller, 2004; Van Vugt, Furuya, Vauth, Jabusch \& Altenmüller, 2014). Así, por ejemplo, como lo menciona Sloboda (1985), la repetición es un factor importante para la adquisición de una habilidad, así como el tiempo que una persona demora en realizar una tarea determinada es un precursor del nivel de habilidad. A su vez, la retroalimentación oportuna permite suprimir aquellos procedimientos que puedan llevar a fallas repetidas, en favor de mantener aquellos que realmente sean exitosos. Por lo anterior, es altamente deseable que los estudiantes puedan practicar repetidas veces sus ejercicios y reciban comentarios valiosos sobre cómo mejorar su desempeño. Además, la posibilidad de recibir retroalimentación fuera del aula va fuertemente en línea con la teoría de la autodeterminación (Deci \& Ryan, 1985, 1991, 2000), basada en tres necesidades innatas fundamentales: la necesidad de ser competente, la de relacionarse y la de autonomía. Así, la retroalimentación oportuna podría aumentar la motivación intrínseca de los estudiantes, según lo planteado por Lambert (2012). Lo anterior pone de manifiesto, tal como lo demuestra Duguet (2014, 2015), que el tipo de práctica pedagógica puede tener un efecto en la motivación y en la manera de estudiar de los y las estudiantes. Desde esta perspectiva, y tal como lo plantea Sadakata, Hoppe, Brandmeyer, Timmers y Desain (2008), la retroalimentación visual en la clase de piano podría ayudar a mejorar la comunicación entre el profesor y los estudiantes, favoreciendo un aprendizaje más efectivo. 


\subsection{Modelos de evaluación de escalas}

En general la literatura presenta diversos trabajos sobre el desarrollo de habilidades motoras de los pianistas (Bangert \& Altenmüller, 2003; Bangert et al., 2006; Pascual-Leone et al., 1995), sin embargo, según nuestro conocimiento y revisión de diversas fuentes, solo en el trabajo de Jabusch y sus colaboradores (Jabusch et al., 2004; Jabusch et al., 2009; Van Vugt et al., 2014) se presenta un modelo de evaluación basado en el análisis de archivos $\mathrm{MIDI}^{2}$. Ahora bien, en este modelo solo se considera la ejecución de una escala de do mayor en 2 octavas, de manera ascendente $\mathrm{y}$ descendente, pero el enfoque principal es determinar el efecto de ciertas condiciones fisiológicas en la interpretación pianística, así como la búsqueda de predictores de la adquisición de competencias motoras en niños y adultos. En base al trabajo de Jabush y otros se tiene también, por ejemplo, el modelo propuesto por Molin (2009).

Si bien estos modelos deben ser considerados en el desarrollo de PianoEF, es necesario destacar que en nuestro trabajo se busca como objetivo primordial el retroalimentar al estudiante de piano según la calidad de su ejecución. Por otro lado, en cuanto a productos tecnológicos, y en base a nuestra búsqueda bibliográfica, no se han encontrado herramientas tecnológicas enfocadas a la solución del problema de retroalimentación descrito. Podemos, sin embargo, citar el trabajo de Riley (2007), quien evalúa, en un estudio de caso, el efecto de distintos tipos de retroalimentación: auditiva, visual (piano roll) y audiovisual, en el aprendizaje de escalas. El estudio fue realizado en dos sesiones: en la primera los estudiantes graban 10 a 15 secuencias de escalas de do mayor, y en la segunda (de retroalimentación) visualizan los videos y escuchan las grabaciones para luego grabar nuevamente las escalas, mejorando las versiones iniciales. El autor muestra que los estudiantes que bajan la mano al pulsar las teclas con el pulgar tienden a aumentar la intensidad. Por otra parte, aquellos estudiantes que mantienen la mano cerca de las teclas tienden a presentar mayor solapamiento entre las notas en comparación con aquellos que mantienen los dedos levantados y curvos, quienes presentan menos solapamiento.

Si bien existen diversos softwares que muestran visualmente cómo tocar una canción, por ejemplo, iluminando las teclas en un teclado virtual, su enfoque es más bien orientado al entretenimiento y no al desarrollo en un contexto de enseñanza formal. Este es precisamente uno de los desafíos que se plantea PianoEF. En efecto, y tal como lo expresan Nijs y Leman (2014), insertar tecnología en prácticas didácticas ya existentes puede hacer dichas prácticas más eficientes, lo que podría tener un efecto importante en los resultados de aprendizaje.

${ }^{2}$ Musical Instrument Digital Interface. 


\subsection{Tecnologías MIDI}

Los primeros antecedentes de la tecnología MIDI aparecen alrededor de 1860 cuando Hermann von Helmholtz, pionero en la utilización de la electrónica para la generación de sonidos, construye una serie de generadores electromecánicos capaces de producir tonos puros. Estos desarrollos se vieron potenciados en 1897 por Thaddeus Cahill, quien construye el Dynamophone o Telharmonium, el primer sintetizador de sonido que permite producir música transmitible eléctricamente por cable. Ya en el año 1961, Harald Bode crea el Melochord, el primer sintetizador controlado por voltaje. En 1964 Robert Moog y Donald Buchla presentan de forma independiente una primera versión de un sintetizador comercial. Varios años después, en 1982, se produce un consenso entre distintos fabricantes y compañías para la definición de lo que hoy se conoce como protocolo MIDI, lo que abre una poderosa conexión entre el mundo digital y computacional y el mundo sonoro.

Un instrumento con sistema MIDI recibe y procesa mensajes en tiempo real. El mensaje más básico es Note $O N$, con el cual se inicia la reproducción de una nota. Por otro lado, el mensaje Note OFF finaliza la reproducción de la nota correspondiente. El protocolo MIDI tiene gran variedad de mensajes y parámetros, entre los que se destacan: (1) el offset: indica el tiempo en milisegundos en que se genera el mensaje MIDI, en base a un valor inicial 0 cuando comienza la grabación del archivo; (2) el pitch o nota involucrada: corresponde a alguna de las notas de la escala occidental tradicional, pudiendo incluir alteraciones de sostenidos o bemoles. Es un valor entero entre 0 y 127, donde el pitch 60 corresponde al DO central. Esta división genera 11 octavas o divisiones de 12 notas, consideradas entre la octava -1 y la octava 9. Esto permite diferenciar las notas indicando la octava a la que pertenecen. Entonces, todas las notas posibles de representar en MIDI corresponden al rango: DO -1, RE -1, ..., DO 4, RE 4, ... SI 9; (3) el canal: un archivo MIDI estándar tiene 16 canales diferentes, que pueden ser asignados cada uno a un instrumento diferente. Esto permite la creación de piezas compuestas que combinan distintos elementos armónicos y melódicos, y (4) la velocidad: corresponde a un valor entero entre 0 y 127 y representa la "fuerza" o "volumen" con el que se presiona o se debe reproducir la nota. Por tanto, un archivo MIDI puede concebirse computacionalmente como un listado secuencial de mensajes con forma: (offset, mensaje, canal, pitch, velocity).

\subsection{Análisis de información MIDI}

Para los propósitos de PianoEF es necesario tener una representación de más alto nivel para analizar archivos MIDI. Para ello, hemos utilizado el lenguaje de programación Python y la librería 
especializada MIDO. Ambas tecnologías son gratuitas y disponibles libremente (https://www.python.org/ y https://github.com/mido/mido). Así, para todos los propósitos de PianoEF concebimos un archivo MIDI como una matriz de notas, tal como se muestra en la Tabla 1. La matriz está compuesta por 4 columnas: pitch, velocity, onset_sec, duration_sec, que representan, respectivamente, la nota tocada, la intensidad, el tiempo en que se toca la nota y su duración. La información temporal está indicada en segundos. Cabe destacar que esta representación es inspirada por la herramienta MIDI Toolbox (Toiviainen \& Eerola, 2016), disponible para el ambiente MATLAB.

Tabla 1

Matriz de notas utilizada como representación de archivo MIDI. La tabla muestra la ejecución de las primeras notas de la escala de do mayor, ascendente, con dos manos.

\begin{tabular}{lllll}
\hline & Pitch & Velocity & Onset_sec & Duration_sec \\
\hline 0 & 60.0 & 72.0 & 2.158854 & 0.945312 \\
1 & 48.0 & 59.0 & 2.174479 & 0.945312 \\
3 & 62.0 & 77.0 & 3.088542 & 0.914062 \\
4 & 50.0 & 67.0 & 3.088542 & 0.869792 \\
5 & 52.0 & 59.0 & 3.942708 & 0.869792 \\
6 & 64.0 & 61.0 & 3.942708 & 0.825521 \\
7 & 65.0 & 64.0 & 4.799479 & 0.838542 \\
8 & 53.0 & 58.0 & 4.812500 & 0.812500 \\
9 & 67.0 & 81.0 & 5.625000 & 0.869792 \\
10 & 55.0 & 64.0 & 5.0638021 & 0.841146 \\
11 & 69.0 & 67.0 & 6.447917 & 0.869792 \\
\hline
\end{tabular}

Fuente: Elaboración propia.

\subsection{PianoEF}

PianoEF se concibe como una plataforma tecnológica para el apoyo al aprendizaje de escalas en el piano, con un especial énfasis en fomentar la autonomía de los estudiantes mediante la entrega de retroalimentación multimodal (i.e., visual, verbal y auditiva).

El principal escenario de uso que se propone para PianoEF es como sigue: (1) el estudiante conecta un piano o teclado al computador, utilizando tecnología MIDI, por ejemplo, mediante interfaces USB-MIDI, disponibles en el comercio; (2) al ingresar a PianoEF, el estudiante indica la escala a ejecutar y sus parámetros de evaluación, tales como articulaciones y otros; (3) luego de 
la ejecución del estudiante, PianoEF presenta al estudiante una evaluación indicando valores cuantitativos, así como un comentario general sobre aspectos a mejorar. La plataforma entrega tres tipos de información (correctitud, articulación/legato e intensidad), lo que se realiza con distintos focos (explícito y no explícito). Con retroalimentación explícita nos referimos a aquella que intenta reforzar normas (Wilson, Lee, Callaghan \& Thorpe, 2008), por ejemplo, la pestaña de articulación, la de correctitud (notas) o la pestaña de grabación de la escala realizada por el profesor. Por su parte, la retroalimentación no explícita se basa en una información de referencia (i.e., pestaña de intensidad). Cabe señalar que la plataforma permite escuchar la grabación de una escala ejecutada por un profesor de la asignatura (modelo explícito) y está diseñada para entregar retroalimentación auditiva mediante registro de audio MIDI que cada usuario graba al momento de ejecutar una nueva versión de la escala.

\subsection{Correctitud de notas tocadas}

El primer análisis corresponde a la correctitud de las notas tocadas. Esto significa que es necesario saber si se tocaron todas las notas de la escala, en el orden adecuado, y también determinar si no se ha presionado ninguna otra nota que no está en la escala. Esto se realiza inspeccionando la matriz de notas correspondiente al archivo cargado por el estudiante, y haciendo una comparación con la matriz de notas esperadas, que ya ha sido precargada en el sistema. Para este primer análisis basta con examinar la columna pitch y determinar si está o no la secuencia de notas esperada. Por ejemplo, la Figura 1 muestra la ejecución correcta de una escala de do mayor. Se observa que PianoEF muestra, en clave americana, las notas esperadas de una ejecución correcta versus las notas efectivamente tocadas por el estudiante. 


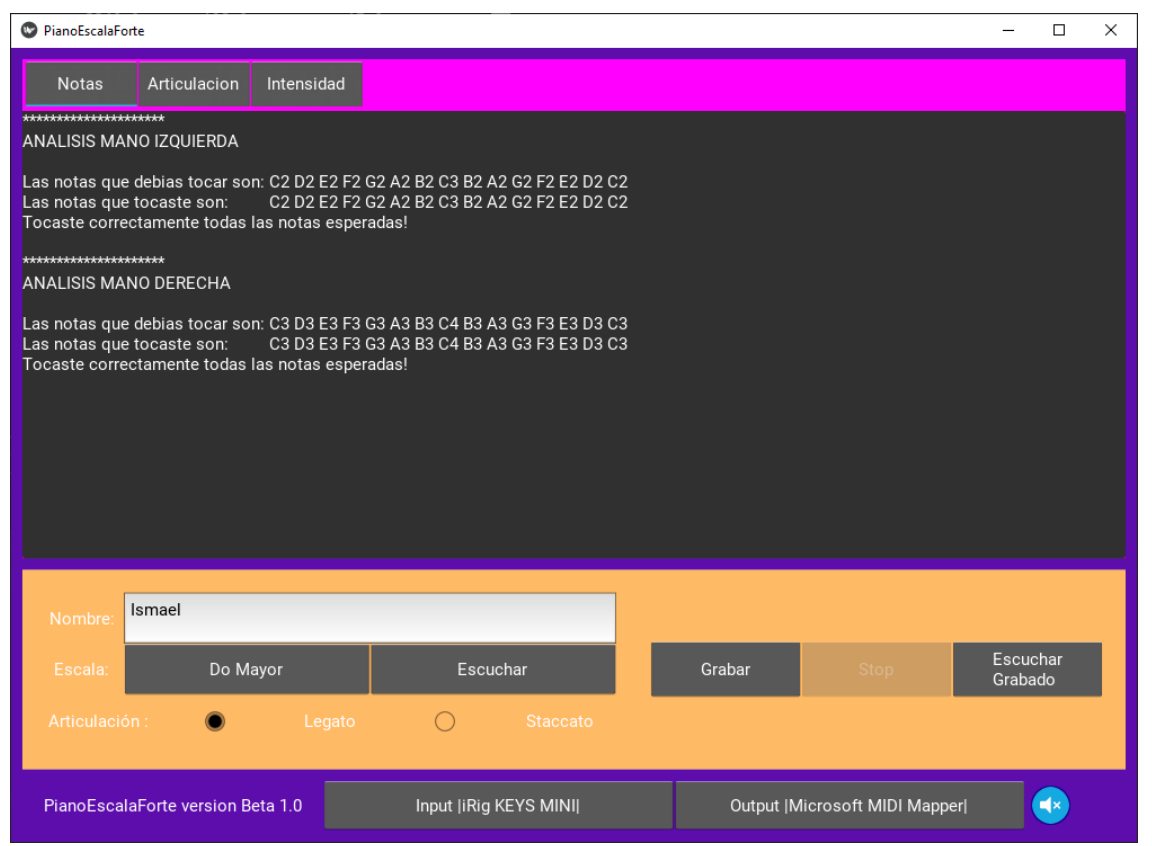

Figura 1. Análisis de ejecución escala do mayor con legato.

Fuente: Elaboración propia.

\subsection{Análisis de articulación e intensidad}

Considerando nuevamente la escala de do mayor con articulación legato, podemos ver en la

Figura 2 la retroalimentación entregada por PianoEF. Aquí se observa que entre algunas notas consecutivas sí hubo legato, pero en otras no; una funcionalidad similar ocurre para el staccato. 


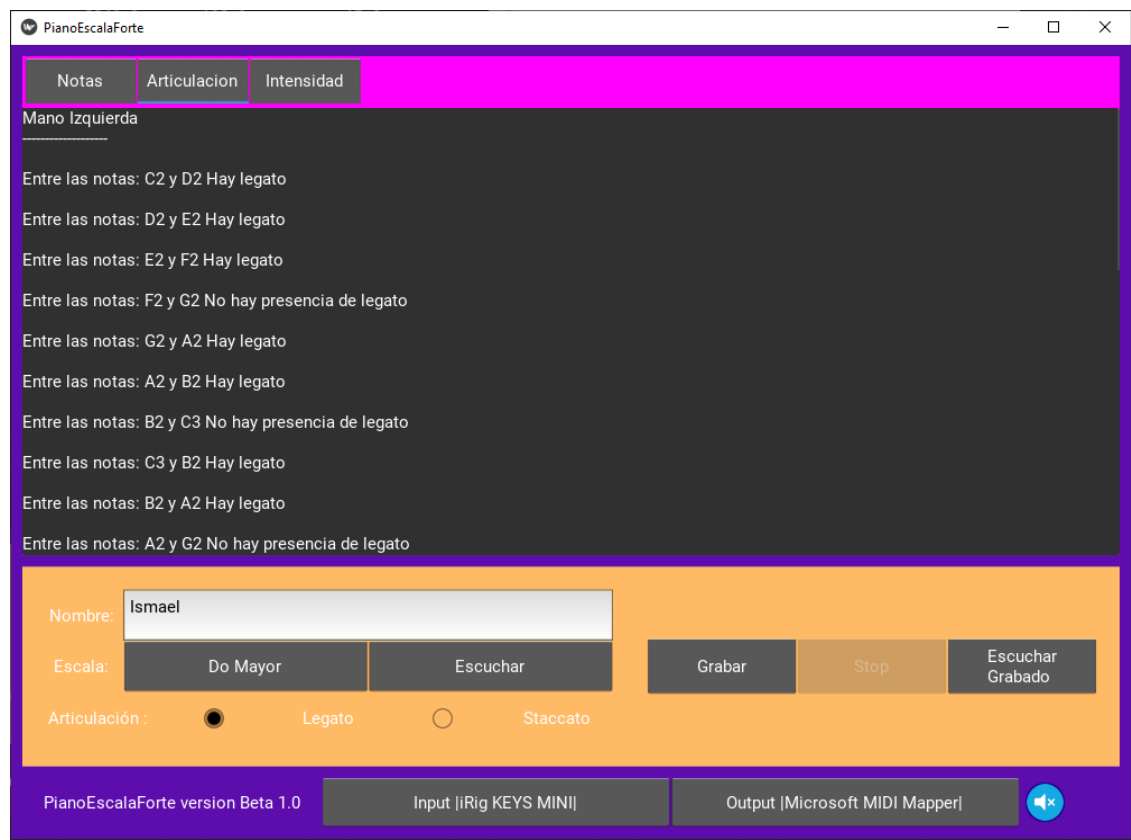

Figura 2. Análisis de articulación legato para la escala de do mayor tocada. PianoEF retroalimenta nota por nota la ocurrencia de esta articulación.

Fuente: Elaboración propia.

También, en la Figura 3 se muestran los gráficos de intensidad, de forma que el estudiante pueda verificar la efectividad de pasajes crescendo o diminuendo. Es importante destacar que todos los análisis se desprenden del análisis de la matriz de notas, y que, por supuesto, esto puede extenderse a otro tipo de verificaciones. Por ejemplo, el presente trabajo se propone estudiar los cambios de intensidad asociados al paso del pulgar durante la ejecución de escalas. En efecto, a nuestro conocimiento, no existe evidencia de su estudio particularmente en estudiantes de primer año de piano. 


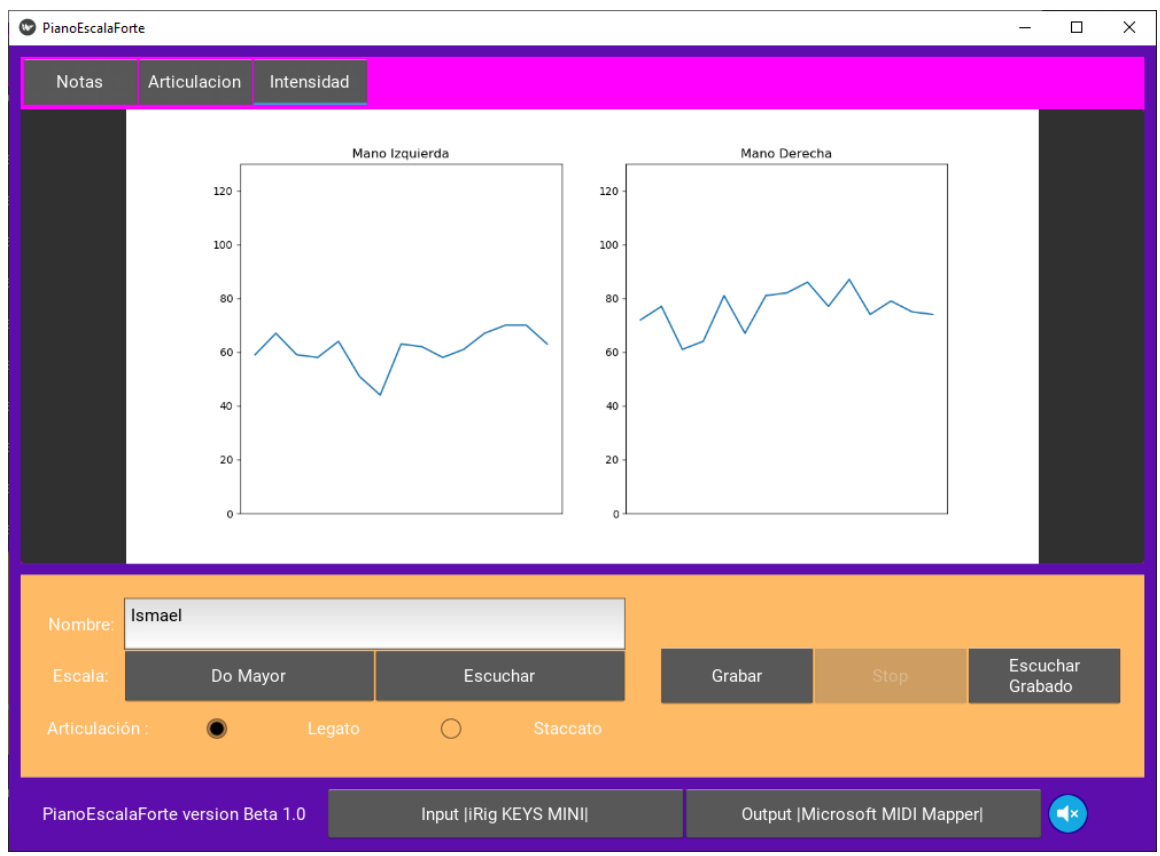

Figura 3. Retroalimentación de la intensidad de las notas de la escala en ambas manos.

Fuente: Elaboración propia.

\subsection{Cálculo de parámetros para el legato}

Para elaborar un algoritmo para evaluar el legato es necesario calcular un solapamiento (óptimo) entre una nota y la que le sigue a continuación. Para lo anterior se procedió a realizar un análisis cuantitativo de ejecuciones de pianistas profesionales, en este caso profesores de la cátedra de piano, en búsqueda de criterios más objetivos y basados en el desempeño de expertos, incluyendo el cálculo de estadísticas (por ejemplo, desviación estándar). Lo anterior nos permitió calcular un rango promedio de solapamiento necesario para considerar un legato como óptimo. Para el estudio y creación de esta plataforma, se calculó que el promedio de tiempo de solapamiento entre dos notas de los profesores era de 0,3 segundos, por lo tanto, si el tiempo de solapamiento es mayor a 0,3 segundos no se considera como legato.

A su vez, en caso de que el tiempo de solapamiento sea inferior a 0,01 segundos no se considera igualmente como legato, es decir, no existe solapamiento entre las notas. Tomando como ejemplo la escala de do mayor, podemos calcular el intervalo de solapamiento entre la nota do (primer grado de la escala de do M) y la nota re (segundo grado de la escala de do mayor) de la siguiente manera: si la nota do es soltada luego de 2,31 segundos de ser pulsada y la nota re es pulsada en el segundo 2,2, restando ambos valores nos da 0,11 segundos $(2,31-2,2=0,11)$. Si el valor es positivo, nos indica que existe solapamiento (Figura 4). 


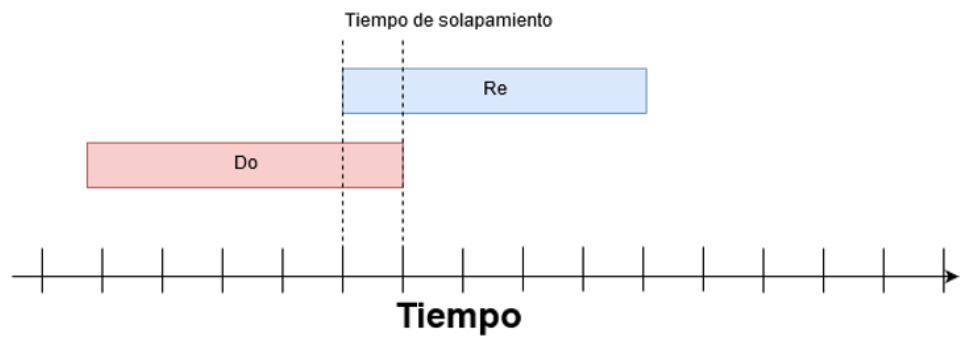

Figura 4. Representación del solapamiento entre dos notas que establece el criterio de definición de la calidad del legato.

Fuente: Elaboración propia.

\section{Método}

Se utiliza un diseño pre-test/post-test que permite evaluar el efecto de la utilización de la plataforma PianoEF en el aprendizaje y perfeccionamiento de una escala musical por medio de una fase de exploración multimodal breve. La investigación es de enfoque mixto. Los datos cuantitativos provienen de la performance de los estudiantes, es decir, los archivos MIDI grabados por PianoEF; mientras que los datos cualitativos son generados luego de la aplicación de un cuestionario una vez finalizada la sesión de evaluación. El objetivo del cuestionario es: (1) determinar cuál es el tipo de retroalimentación más efectiva para los estudiantes, en cuanto a la información desplegada y la manera de visualizarla; (2) determinar si la retroalimentación otorgada por PianoEF efectivamente mejora el desempeño de los estudiantes; (3) obtener información acerca de las estrategias de estudio de los participantes, y (4) evaluar la eficacia de la plataforma PianoEF en el contexto de la clase de piano grupal, en la cual participan entre 4 y 7 estudiantes. Dado este carácter grupal, se hace necesario el trabajo con pianos electrónicos y audífonos individuales.

Cabe señalar que el estudio de las escalas en el contexto de estudio se realiza desde el inicio del curso y le permite al estudiante un primer acercamiento a la ejecución pianística. Los estudiantes comienzan tocando el pentacordio, es decir, un grupo de 5 notas (5 dedos), que se ejecutan de manera simultánea y luego, de manera consecutiva. Lo anterior permite adquirir el control del sonido a partir de la posición de la mano para lograr un manejo de los distintos parámetros musicales que intervienen en la interpretación (regularidad, disociación, intensidad y aplicación de articulaciones). El segundo paso es ir estudiando gradualmente las distintas escalas previstas en el programa de estudio. Las escalas se abordan de forma teórica y práctica, desde su construcción y comprensión, hasta sus distintas aplicaciones en el teclado. Se comienza con la escala de do mayor y luego se van agregando de manera paulatina las demás tonalidades según sea su complejidad en el mundo tonal. Las escalas que se enseñan en primer año son las siguientes: do $M$, sol M, re $M$, fa $M$ y sus relativas menores (la $m$, mi m, si m, re m). 
A continuación, se detalla la configuración y metodología de estas sesiones, y en la siguiente sección se presentan y discuten los resultados.

\subsection{Participantes}

Se contó con la participación de 14 estudiantes de primer año de piano que asisten a clases de piano grupal en el Instituto de Música de la Pontificia Universidad Católica de Valparaíso (PUCV). Los estudiantes provienen de las carreras de Licenciatura en Música, Pedagogía en Música e Interpretación Musical. Su edad promedio es de 22,13 años (DS = 2,7). Solo dos estudiantes declaran haber tenido un año de clases formales de piano antes de iniciar el curso de piano grupal.

\subsection{Equipamiento}

Cada participante utilizó una Clavinova de marca Yamaha modelo CLP230, además de una interfaz MIDI de marca Roland UM-ONE MK2 conectada a la Clavinova hacia un notebook con la aplicación instalada.

\subsection{Instrumentos de toma de información}

Se elaboró un cuestionario dividido en dos secciones. La primera está destinada a recoger datos sociodemográficos de los participantes y las estrategias que utilizan (si las tienen) para el estudio de las escalas. Se plantean dos preguntas abiertas, la primera se refiere a las estrategias utilizadas por los estudiantes para el estudio de escalas ("Describa de qué manera estudia habitualmente las escalas en el piano") y la segunda se interesa en las dificultades encontradas en su práctica ("Señale las dificultades que usted ha tenido para el aprendizaje de escalas en el piano"). La segunda sección se destina a evaluar la plataforma PianoEF en sus dimensiones técnicas y pedagógicas, para lo cual se utilizan preguntas abiertas y cerradas. Las preguntas abiertas consultan sobre la eficacia del tipo de retroalimentación ("Describa qué tipo de retroalimentación, verbal, gráfica o audio, le permitió mejorar significativamente su ejecución de escalas") y sobre las estrategias de estudio utilizadas durante la sesión para el estudio de las escalas. Las preguntas cerradas son de tipo Likert y evalúan si: (1) las actividades realizadas son importantes y contribuyen al aprendizaje; (2) el tamaño y tipo de letra de la plataforma es claro 
y legible; (3) el uso de la plataforma permite mejorar la ejecución de escalas, y (4) este tipo de dispositivos podría contribuir al desarrollo de la clase de piano grupal.

\subsection{Análisis de datos}

A partir de las preguntas abiertas del cuestionario se codifican conceptos e ideas clave relacionados con los temas tratados. Se analiza la información y se elaboran categorías. Cabe señalar que, en la pregunta referida a las modalidades de retroalimentación, la construcción de categorías es menos flexible ya que se espera que las respuestas se ajusten a los tipos de retroalimentación estudiados. No ocurre lo mismo con la pregunta referida a las estrategias de estudio durante la sesión. Para cumplir con lo anterior se realiza una codificación abierta y simultáneamente una codificación axial (Strauss \& Corbin, 1990). La codificación está destinada a "identificar los conceptos, dimensiones y construir categorías y subcategorías pertinentes a la investigación” (Quilaqueo \& San Martin, 2008, p. 155). La codificación axial, por su parte, implica relacionar categorías con subcategorías, con el objetivo de ir aclarando el fenómeno estudiado (Strauss \& Corbin, 1990). Para comprobar estadísticamente si existen diferencias entre la fase pre-test y post-test, se aplican pruebas $T$ de Student en el conjunto de variables estudiadas.

\subsection{Procedimiento}

La presente investigación se realiza en dos etapas, la primera consiste en el desarrollo de la plataforma, el que ha sido descrito en las secciones anteriores. Es importante destacar que los distintos parámetros de legato, articulación e intensidad fueron generados a partir de grabaciones en formato MIDI realizadas por los distintos profesores de la cátedra de piano del Instituto de Música de la PUCV.

La segunda etapa se divide en dos sesiones, la primera de familiarización con la plataforma y la segunda de evaluación. La sesión de familiarización tiene una duración de aproximadamente 30 minutos y es conducida una semana antes de la sesión de evaluación por el equipo de investigadores. Los estudiantes realizan ejercicios con la plataforma conociendo de esta manera sus diferentes pestañas y sus respectivas funciones. El equipo investigador resuelve dudas y responde consultas sobre la utilización de la plataforma.

La fase de evaluación, por su parte, consiste en una breve sesión de aproximadamente 20 minutos destinada a la utilización de la plataforma PianoEF para el aprendizaje de una nueva 
escala y tiene lugar al inicio de una clase de piano grupal. Se trabaja con la escala de do mayor, con manos paralelas, ascendente y descendente. Antes de iniciar la sesión de evaluación, se explica a los estudiantes la digitación que deben usar. El protocolo de evaluación tiene una duración de aproximadamente 20 minutos y se divide en tres etapas: (1) ejecución inicial de la escala, donde los estudiantes deben grabar, utilizando la plataforma, una primera versión de la escala (pre-test); (2) fase de exploración, donde pueden libremente examinar las distintas alternativas de visualización de la información disponibles en las pestañas de la plataforma PianoEF (i.e., de correctitud, articulación, legato e intensidad, grabación, audición de la escala grabada y audición de la escala grabada por un docente) y realizar distintas pruebas con la finalidad de mejorar la ejecución gracias a la retroalimentación otorgada por el sistema, y (3) grabación de la escala en su versión final, en donde los estudiantes toman en consideración la retroalimentación obtenida (post-test), para luego responder en una hoja aparte el cuestionario.

\section{Resultados}

\subsection{Análisis del desempeño}

Se compararon las ejecuciones de la escala de do mayor realizadas en una sesión antes del uso de la plataforma PianoEF y después de su uso. En general los estudiantes conocían y ejecutaban correctamente las notas de la escala, por lo tanto, el trabajo se concentró esencialmente en dos aspectos: (1) uniformidad de la intensidad, y (2) aplicación correcta de legato. Se apreció una mejora respecto a la regularidad en la intensidad con la que los estudiantes tocaban la escala. Se calculó la desviación estándar de la intensidad (rango 0-127) con la cual se ejecutó cada nota de la escala. En promedio los participantes obtuvieron una mejora del $15 \%(\mathrm{M}=59, \mathrm{DS}=8,33$ a $\mathrm{M}$ $=58,31, D S=7,14)$, y un estudiante en particular mejoró en un $60 \%(M=52, D S=13,56$ a $M=$ $61, D S=8,46)$, manteniendo un mejor control durante la ejecución de la escala. Además, se calculó el solapamiento de las notas ejecutadas para evaluar la calidad del legato. Después de algunos minutos de práctica, los estudiantes lograron perfeccionar el legato en la escala de do mayor disminuyendo el solapamiento entre las notas y su variabilidad, sin embargo, la diferencia no fue significativa entre ambas fases de práctica. En paralelo analizamos la intensidad asociada al paso del pulgar. En efecto se observa que durante el paso del pulgar se produce un cambio en la intensidad con relación a la registrada en los otros dedos. Gracias al uso de la plataforma, los estudiantes lograron incrementar en un $27 \%$ la intensidad con la que tocaban la nota correspondiente al traspaso del pulgar, logrando estar más cerca de la media y manteniendo una mayor uniformidad respecto a la intensidad con la que tocaban la escala en general. Respecto al tempo, no se observaron diferencias significativas entre la fase inicial y la post-estudio (Tabla 2). 
Cabe destacar que en ambos indicadores de variabilidad de la intensidad (Int PS DS; Int SP DS) la magnitud del efecto es alto (Cohen, 1988).

Tabla 2

Comparación entre las distintas variables estudiadas antes y después de la fase de práctica con la plataforma

\begin{tabular}{|c|c|c|c|c|c|c|c|c|c|}
\hline \multirow[b]{2}{*}{ Variable } & \multicolumn{2}{|l|}{ Antes } & \multicolumn{2}{|c|}{ Después } & \multirow[b]{2}{*}{$t(9)$} & \multirow[b]{2}{*}{$p$} & \multicolumn{2}{|c|}{$95 \%$ IC } & \multirow{2}{*}{$\begin{array}{c}\text { Cohen } \\
\qquad d\end{array}$} \\
\hline & $M$ & $D S$ & $M$ & $D S$ & & & $L I$ & $L S$ & \\
\hline Tempo & 71,78 & 23,43 & 79 & 36,29 &,- 84 & ,41 & $-25,6$ & 11,18 & 0,24 \\
\hline Inten PS & 55,91 & 12,89 & 56,6 & 12,57 &,- 28 & ,78 & $-5,95$ & 4,58 & 0,05 \\
\hline Int $S P$ & 58,93 & 11,91 & 59,14 & 15,16 &,- 09 & ,93 & $-5,65$ & 5,22 & 0,02 \\
\hline Int PS DS & 8,33 & 2,32 & 7,18 & 1,06 & 2,22 & ,045 & ,03 & 2,27 & 0,64 \\
\hline Int SP DS & 8,95 & 5,3 & 6,52 & 2,7 & 1,85 & ,088 &,- 41 & 5,27 & 0,58 \\
\hline Legato & 1,04 & ,31 & ,99 &, 48 &, 59 &, 57 &,- 15 & ,26 & 0,12 \\
\hline LegatoDS & ,26 & 15 & ,24 & ,27 & ,27 & ,78 &,- 11 & ,14 & 0,09 \\
\hline
\end{tabular}

Nota: IC = intervalo de confianza (95\%); LI = límite inferior; $L S=$ límite superior; Int PS = intensidad en el paso del pulgar; Int SP = intensidad sin considerar el paso del pulgar; Int PS DS = desviación estándar del paso del pulgar; Int SP DS = desviación estándar sin considerar el paso del pulgar; Legato DS = desviación estándar del legato.

\subsection{Cuestionarios}

La información se obtuvo de preguntas cerradas y abiertas presentes en el cuestionario aplicado a los estudiantes al final de la sesión de evaluación. Se analizaron las representaciones discursivas de los estudiantes con el fin de elaborar categorías significativas. Resultaron como categorías representativas aquellas que abordaban aspectos relacionados con las modalidades de aprendizaje (i.e., visual, auditivo, verbal) tal como lo indicaba la pregunta 1 ("Describa qué tipo de retroalimentación -verbal, gráfica, audio- le permitió mejorar significativamente su ejecución de escalas. Si no fuese el caso, describa por qué"). La información se presenta en la Tabla 3. 


\section{Tabla 3}

Ejemplos de verbalizaciones asociadas a las distintas modalidades de retroalimentación que entrega la plataforma PianoEF

\begin{tabular}{ll}
\hline Modalidad & Unidades de sentido \\
\hline Audio & Escuchar el audio de muestra, aunque por tener poca \\
& experiencia me faltó práctica \\
& \\
& Escucharse... cómo fue mi desempeño da una retroalimentación (E17) \\
& importante
\end{tabular}

Gráfica

La gráfica, ya que mediante esta me di cuenta de mi falta de

precisión para tocar las notas

El gráfico ayudó a repasar los errores que cometía

Me permitió ir arreglando la ejecución de ciertas notas, la

intensidad igual fue de gran ayuda

Esquema gráfico, debido a que buscaba igualdades en el peso y

no tensar cada dedo en cada nota

Me ayudó a ver que el peso de mis dedos era muy disparejo

Verbal

La descripción de las articulaciones, que no realicé bien... me

permitió tomar conciencia para mejorar

La información de legato no me fue tan útil en primera instancia

La retroalimentación más útil fue la de articulación, ya que

percibe con gran detalle cuándo un legato era ejecutado correctamente o no

Fuente: Elaboración propia.

Con relación a las estrategias de estudio que los estudiantes utilizaron durante la sesión de evaluación (pregunta 2), las categorías representativas reflejan los distintos procedimientos y procesos cognitivos que emergen de la interacción con la plataforma como parte de un proceso continuo de incorporación tecnológica (Tabla 4). 


\section{Tabla 4}

Categorías obtenidas luego de realizar una codificación axial con los datos provenientes del cuestionario

\begin{tabular}{|c|c|c|}
\hline Categorías & Unidades de sentido & \\
\hline Operativa & $\begin{array}{l}\text { Tocaba y me fijaba en el gráfico para saber si la intensidad al } \\
\text { pulsar las teclas era la correcta o no, y también saber qué notas } \\
\text { no ligaba }\end{array}$ & (E6) \\
\hline \multirow[t]{3}{*}{ Repetición } & Repetir y fijarme en las intensidades & $(\mathrm{E} 1)$ \\
\hline & Repetir la moción de los dedos lentamente & (E4) \\
\hline & Solo repetí la escala hasta que me sentí conforme & (E14) \\
\hline Corrrectitud & $\begin{array}{l}\text { Observar la intensidad, si las notas estaban correctamente } \\
\text { ejecutadas }\end{array}$ & $(\mathrm{E} 2)$ \\
\hline \multirow[t]{3}{*}{ Legato } & $\begin{array}{l}\text { Miraba los datos de la plataforma para notar en qué notas no } \\
\text { estaba ligando }\end{array}$ & (E3) \\
\hline & Intenté enlazar mejor las notas para lograr el legato & (E5) \\
\hline & $\begin{array}{l}\text { Tocar reiteradas veces y lograr un legato completo y un peso } \\
\text { uniforme }\end{array}$ & (E11) \\
\hline Articulación & $\begin{array}{l}\text { Intenté mejorar los problemas de anticipación que demandaba } \\
\text { el programa, aplicando peso correspondiente }\end{array}$ & (E7) \\
\hline \multirow[t]{2}{*}{ Propiocepción } & Calcular el peso de la mano & (E8) \\
\hline & $\begin{array}{l}\text { Controlar el peso de cada dedo en la ejecución de las notas, al } \\
\text { revisar los estudios me daba cuenta de cuál era la mano que } \\
\text { más tensaba }\end{array}$ & (E9) \\
\hline $\begin{array}{l}\text { Manos } \\
\text { separadas }\end{array}$ & $\begin{array}{l}\text { Estudiar por separado, juntar las } 2 \text { manos en movimiento } \\
\text { paralelo y contrario }\end{array}$ & (E10) \\
\hline Completa & $\begin{array}{l}\text { Escuchar la grabación de la ejecución, además de ver las } \\
\text { articulaciones y gráficos de intensidad }\end{array}$ & (E12) \\
\hline
\end{tabular}

Fuente: Elaboración propia.

En lo que respecta a las preguntas cerradas (escala graduada tipo Likert) se realizó una prueba de fiabilidad que permitió determinar distintas dimensiones de análisis. Al considerar las 4 
sentencias se obtiene un alfa de Cronbach $=.69$. Considerando las sentencias 1 y 4 , se obtiene un alfa de Cronbach $=.85$. Si consideramos las sentencias 1,3 y 4 el alfa de es de Cronbach $=.75$. Lo anterior es lógico si consideramos que la sentencia 2 se relaciona directamente con aspectos referidos a la presentación visual de la plataforma. La sentencia 3, por su parte, se relaciona tanto con la actividad realizada en la misma sesión como con la estimación de su eficacia como herramienta de aprendizaje (Tabla 5).

\section{Tabla 5}

Estadísticas descriptivas y resultados por tipo de pregunta y categorizadas por cuartil

\begin{tabular}{lllllll}
\hline & Media & Moda & DS & P25 & P50 & P75 \\
\hline Sentencia 1 & 4,43 & 5 & 0,79 & 4 & 5 & 5 \\
Sentencia 2 & 4 & 4 & 0,85 & 4 & 4 & 5 \\
Sentencia 3 & 4,04 & 4 & 0,98 & 4 & 4 & 5 \\
Sentencia 4 & 4,35 & 5 & 1,03 & 4 & 5 & 5
\end{tabular}

Nota. DS = desviación estándar; P25 = percentil 25; P50 = percentil 50; P75 = percentil 75; Sentencia 1 = Las actividades realizadas son importantes y contribuyen al aprendizaje; Sentencia 2 = El tamaño y tipo de letra de la plataforma es claro y legible; Sentencia 3 = El uso de la plataforma permite mejorar la ejecución de escalas; Sentencia 4 = ¿Este tipo de dispositivos podría contribuir al desarrollo de la clase de piano grupal?

Fuente: Elaboración propia.

\subsubsection{Estrategias y dificultades en el estudio de las escalas}

Se analizaron las respuestas de los y las estudiantes relacionadas con el estudio personal que realizan a diario. A continuación, se mencionan las distintas estrategias utilizadas por los diferentes grupos que asisten a la clase de piano grupal, así como las dificultades que han experimentado en el aprendizaje de las escalas.

Respecto a las estrategias de estudio de escalas, los estudiantes declaran que al practicar las escalas: utilizan ambas manos, repiten los ejercicios, realizan movimientos paralelos y contrarios, incluyen dedajes y articulación legato, realizan ejercicios de digitación y disociación, así como movimientos paralelos ascendentes y descendentes. Por su parte, los estudiantes de Interpretación tocan generalmente las escalas en tonalidades mayores de forma paralela o contraria, y con metrónomo y muy lento.

Con relación a las dificultades que han experimentado los estudiantes respecto al aprendizaje de las escalas, estos declaran que tienen dificultades: para coordinar ambas manos, con la posición de los dedos en escalas con sostenidos, para pensar cada mano individualmente, para encontrar un ritmo que les permita practicar la fluidez y la disociación/coordinación de los dedos, y con la 
digitación o la disociación. Por su parte, los estudiantes de Interpretación tienen principalmente dificultades con la mano izquierda (sobretodo el dedo 4 debido a su poca agilidad).

\section{Conclusiones}

En este artículo hemos presentado PianoEF, una plataforma tecnológica para el apoyo en el aprendizaje de escalas en el piano. Se ha mostrado cómo funciona el primer prototipo de esta plataforma, implementado como una aplicación de escritorio para plataforma Windows. PianoEF le permite al estudiante, en su período personal de estudio, atender exhaustivamente los distintos parámetros presentes en su ejecución, tales como la regularidad del pulso, es decir, la igual distancia temporal entre un evento sonoro (la nota musical) y otro; la relación de intensidad entre notas (por ejemplo, creciente, decreciente o igual), y la correcta lectura de las notas musicales según lo indicado en la partitura. Se ha demostrado que PianoEF es una herramienta pertinente para ser utilizada en el contexto de la clase de piano grupal y en protocolos de microaprendizaje.

Dentro de los análisis realizados por PianoEF hemos abordado el análisis de la precisión de la ejecución (errores) y de articulaciones específicas como el legato y la intensidad, utilizando criterios cuantitativos y cualitativos. Lo anterior, con la finalidad de conocer cómo PianoEF es percibido por los estudiantes, en cuanto a la calidad de la retroalimentación. El fin último de PianoEF es ser utilizado de manera efectiva en el aula, como apoyo a los procesos pedagógicos en el ámbito de la música, comparada a la de otras disciplinas, puede ser subjetiva. En efecto, PianoEF permitiría, en el contexto de la clase de piano grupal, evitar que exista una percepción errónea o una mala interpretación de la información otorgada por el profesor (Nijs \& Leman, 2014; Welch, Howard, Himonides \& Brereton, 2005).

Los resultados del análisis de la performance muestran un efecto de la utilización de la plataforma (fase de exploración) en la regulación de los parámetros de intensidad. Lo anterior se observa de manera significativa al considerar particularmente los eventos posteriores al paso del pulgar y de manera tendencial al considerar la intensidad de las notas que no se relacionan directamente con el paso del pulgar. En ambos casos el tamaño del efecto es alto, lo que nos permite sugerir que nuestros resultados podrían generalizarse hacia otros contextos de estudio. Considerada la fase de desarrollo de la plataforma y las instrucciones dadas a los participantes, la plataforma PianoEF podría asegurar un control de la intensidad en las primeras fases del aprendizaje de las escalas. Por otra parte, no es sorprendente que los estudiantes no hayan aumentado el tempo de ejecución entre ambas fases de práctica. En efecto, las instrucciones dadas a los participantes no expresaron dicha necesidad. 
Gracias a PianoEf los estudiantes logran identificar los parámetros de la performance que requieren perfeccionar, lo que favorece la metacognición y el desarrollo paralelo de la autonomía (Osses \& Jaramillo, 2008). Los estudiantes, por lo demás, evalúan positivamente su inclusión como herramienta de apoyo al aprendizaje de escalas en el contexto de la clase de piano grupal, lo que logra cubrir un ámbito deficitario planteado en la bibliografía, particularmente lo referido al uso de tecnologías para el apoyo de estudiantes con distintos perfiles de entrada a las carreras universitarias (Tejada \& Pérez-Gil, 2016). Lo anterior es muy común en nuestro contexto de estudio.

En lo que respecta la retroalimentación multimodal que entrega PianoEF, nuestros resultados son consistentes con investigaciones precedentes que muestran que la utilización de biofeedback y de feedback visual son de gran ayuda tanto para los estudiantes que preparan una nueva pieza de música (Riley, 2005) como para aquellos que perfeccionan los aspectos técnicos en la ejecución de escalas en el piano (Riley, 2007), independientemente del nivel de los pianistas. En nuestro estudio, la utilización de feedback multimodal, es decir, visual (intensidad) y verbal (legato) ayuda a los estudiantes a planificar la ejecución de las escalas; dicha fluidez en la preparación de secuencias de movimientos es un indicador esencial de la adquisición de competencia musicales (Drake \& Palmer, 2000). En efecto, la plataforma ayuda al estudiante a tener conciencia de la coordinación de ciertos movimientos necesarios para el control técnico, lo que según Riley et al. (2005) conlleva, desde un punto de vista musical, a una mejora del sonido. Dichos elementos podrían pasar desapercibidos sin la intervención de este tipo de dispositivos. Por lo demás, en nuestra metodología, la fase de exploración parece estimular la flexibilidad y enriquecer los procesos de exploración creativa, lo que según Timmers, Sadakata y Desain (2012) forma parte del desarrollo de habilidades musicales y ayuda a mejorar la performance.

Cabe señalar, sin embargo, que los estudios de Riley $(2005,2007)$ citados previamente no fueron conducidos en el contexto de una clase de piano grupal. Lo anterior abre nuevas perspectivas a nuestro trabajo ya que la plataforma PianoEF está diseñada para ser utilizada en el contexto de una clase de piano grupal. En efecto, hemos observado que la plataforma PianoEF es igualmente eficaz en el aprendizaje de escalas en estudiantes de distintas carreras que cursan dicha metodología de enseñanza (i.e., Interpretación Musical, Licenciatura en Música y Pedagogía en Música). En el caso de los estudiantes de Pedagogía en Música este tipo de dispositivos podría ayudarlos en su proceso de construcción de una identidad profesional (ligada al uso de tecnologías), lo que implicaría un proceso de actualización de metacompetencias. Ese proceso de construcción necesitará una renegociación en la transición de la vida de estudiante al 
momento de afrontar el mercado laboral (Juuti \& Littleton, 2010, 2012; Middleton \& Middleton, 2017), lo que podría estar mediado por competencias relacionadas con el uso de TIC.

Las limitaciones del presente trabajo tienen relación con la utilización de una muestra reducida. Lo anterior está ligado, sin embargo, al número de estudiantes disponibles en las clases de piano grupal en el contexto de estudio. Otra de las limitaciones del presente trabajo es la ausencia de un pre-test/post-test que cubra un intervalo de varias sesiones para poder medir la eficacia en el tiempo de utilización de la plataforma.

Es necesario considerar, finalmente, que la inclusión de tecnologías de la información es un proceso continuo y que debe evaluarse constantemente, lo que puede realizarse, en el ámbito universitario, a través de distintos criterios según niveles progresivos de incorporación tecnológica (Nolasco \& Ojeda, 2016). El presente trabajo contribuye a aportar nuevas visiones sobre el proceso inicial de implementación de TIC en la clase de piano grupal a través del uso de la plataforma PianoEF. 


\section{Referencias Bibliográficas}

Bangert, M., \& Altenmüller, E. O. (2003). Mapping perception to action in piano practice: a longitudinal dc-eeg study. BMC Neuroscience, 4(1), 26. doi:10.1186/1471-2202-4-26

Bangert, M., Peschel, T., Schlaug, G., Rotte, M., Drescher, D., Hinrichs, ... Altenmüller, E. (2006). Shared networks for auditory and motor processing in professional pianists: evidence from fMRI conjunction. Neurolmage, 30, 917-926. doi: 10.1016/j.neuroimage.2005.10.044

Cara, M., \& Aranda, R. (2016). Autoeficacia y transformación dinámica del aprendizaje en la práctica docente inicial. Perspectiva Educacional, 55(1), 3-19. doi:10.4151/07189729Vol.55-Iss.1-Art.359

Ciccolini, A., \& Lafaye, J-J. (1998). Aldo Ciccolini: musique et vérité: entretiens. Paris: Editions du Félin.

Cohen, J. (1988). Statistical power analysis for the behavioral sciences (2a ed.). Hillsdale, NJ: Erlbaum

Deci, E. L., \& Ryan, R. M. (1985). Intrinsic motivation and self-determination in human behavior. New York: Plenum Press.

Deci, E. L., \& Ryan, R. M. (1991). A motivational approach to self: Integration in personality. En R. Dienstbier (Ed.). Nebraska symposium on motivation: Vol. 38. Perspectives on motivation (pp. 237-288). Lincoln: University of Nebraska Press.

Deci, E. L., \& Ryan, R. M. (2000). The "what" and "why" of goal pursuits: Human needs and the self-determination of behavior. Psychological inquiry, 11(4), 227-268. doi:10.1207/S15327965PLI1104_01

De Jong, T. (2010). Cognitive load theory, educational research, and instructional design: some food for thought. Instructional Science, 38(2), 105-134. doi:10.1007/s11251-009$9110-0$

Drake, C., \& Palmer, C. (2000). Skill acquisition in music performance: relations between planning and temporal control. Cognition, 72, 1-33. doi:10.1016/S0010-0277(99)00061-X 
Duguet, A. (2014). Les pratiques pédagogiques en première année universitaire: description et analyse de leurs implications sur la scolarité des étudiants (Tesis Doctoral). Recuperado desde https://tel.archives-ouvertes.fr/tel-01217315/document

Duguet, A. (2015). Les pratiques pédagogiques à l'université en France: quels effets sur la réussite en première année? Le cas du cours magistral. Recherche et formation, 2(79), 9-26.

Jabusch, H. C., Alpers, H., Kopiez, R., Vault, H., \& Altenmüller, E. (2009). The influence of practice on the development of motor skills in pianists: a longitudinal study in a selected motor task. Human Movement Science, 28(1), 74-84. doi:10.1016/j.humov.2008.08.001

Jabusch, H. C., Vauth, H., \& Altenmüller, E. (2004). Quantification of focal dystonia in pianists using scale analysis. Movement Disorders, 19(2), 171-180. doi:10.1002/mds.10671

Juuti, S., \& Littleton, K. (2010). Musical identities in transition: Solo-piano students' accounts of entering the academy. Psychology of Music, 38, 481-497. doi:10.1177/0305735609351915.

Juuti, S., \& Littleton, K. (2012). Tracing the Transition from Study to a Contemporary Creative Working Life: The Trajectories of Professional Musicians. Vocations and Learning, 5(1), 5-21. doi: 10.1007/s12186-011-9062-9

Lambert, M. (2012). La performance académique des étudiants en premiére année universitaire: influence des capacités cognitives et de la motivation. (Tesis Doctoral). Recuperado desde https://tel.archives-ouvertes.fr/tel-00780578v1/document

Middleton, J. C., \& Middleton, J. A. (2017). Review of literature on the career transitions of performing artists pursuing career development. International Journal for Educational and Vocational Guidance, 17(2), 211-232. doi:10.1007/s10775-016- 9326-x

Minder, M. (1996). Didactique fonctionnelle. Objectifs, stratégies, évaluation. Bruxelles: De Boeck Université.

Molin, P. (2009). Quantitative multidimensional approach of technical pianistic level. En A. Williamon, S. Pretty, \& R. Buck (Eds.). Proceedings of ISPS 2009 (pp. 71-76). Utrecht, The Netherlands: European Association of Conservatoires (AEC). Recuperado desde https://www.researchgate.net/profile/Aaron_Williamon/publication/312293711_Pr 
oceedings_of_the_International_Symposium_on_Performance_Science_2009/links/ 5878b44c08ae8fce4932524f/Proceedings-of-the-International-Symposium-onPerformance-Science-2009.pdf

Nijs, L., \& Leman, M. (2014). Interactive technologies in the instrumental music classroom: A longitudinal study with the Music Paint Machine. Computers \& Education, 73(3), 4059. doi:10.1016/j.compedu.2013.11.008

Nolasco, P., \& Ojeda, M. M. (2016). La Evaluación de la Integración de las TIC en la Educación Superior: Fundamento para una Metodología. RED-Revista de Educación a Distancia, 9(48), 1-24. Recuperado desde https://revistas.um.es/red/article/view/253511

Osses, S., \& Jaramillo, S. (2008). Metacognición: Un camino para aprender a aprender. Estudios Pedagógicos, 24(1), 187-198. doi:10.4067/S0718-07052008000100011

Palazón, J. (2015). Aprendizaje móvil basado en microcontenidos como apoyo a la interpretación instrumental en el aula de música en secundaria. Píxel-Bit. Revista de Medios y Educación, 46, 119-136.

Pascual-Leone, A., Dang, N., Cohen, L. G., Brasil-Neto, J., Cammarota, A., \& Hallett, M. (1995). Modulation of motor responses evoked by transcranial magnetic stimulation during the acquisition of new fine motor skills. Journal of Neurophysiology, 74, 1037-1045. doi:10.1152/jn.1995.74.3.1037

Quilaqueo, D., \& San Martín, D. (2008). Categorización de saberes educativos mapuche mediante la teoría fundamentada. Estudios Pedagógicos 34(2), 151-168. doi:10.4067/S071807052008000200009

Riley, K. (2005). Understanding piano playing through MIDI: Students' perspectives on performance analysis and learning. American Music Teacher, 54(6), 33-37.

Riley, K. (2007). New views of piano playing through motion analysis and midi technology. Journal of Technology in Music Learning, 4(1), 17-30.

Riley, K., Coons, E. E., \& Marcarian, D. (2005). The use of multimodal feedback in retraining complex technical skills of piano performance. Medical Problems of Performing Artists, 20(2), 82-88. 
Sadakata, M., Hoppe, D., Brandmeyer, A., Timmers, R., \& Desain, P. (2008). Real-time visual feedback for learning to perform short rhythms with expressive variations in timing and loudness. Journal of New Music Research, 37(3), 207-220. doi:10.1080/09298210802322401

Sloboda, J. A. (1985). L'esprit musicien: la psychologie cognitive de la musique. Liège: Pierre Mardaga.

Strauss, A., \& Corbin, J. (1990). Basics of qualitative research: grounded theory, procedures and techniques. Newbury Park, CA: Sage.

Tejada, J., \& Pérez-Gil, M. (2016). Diseño y evaluación de un programa informático para la educación musical de maestros no especialistas. El caso de EMOLab. Revista Electrónica Complutense de Investigación en Educación Musical, RECIEM, 13, 22-49. doi:10.5209/RECIEM.52072

Timmers, R., Sadakata, M., \& Desain, P. (2012). The Role of Visual Feedback and Creative Exploration for the Improvement of Timing Accuracy in Performing Musical Ornaments. Music Perception, 30(2), 187-204. doi:10.1525/mp.2012.30.2.187

Toiviainen, P., \& Eerola, T. (2016). MIDI toolbox 1.1. Recuperado desde https://github.com/miditoolbox/

Van Vugt, F., Furuya, S., Vauth, H., Jabusch, H-C., \& Altenmüller, E. (2014). Playing beautifully when you have to be fast: spatial and temporal symmetries of movement patterns in skilled piano performance at different tempi. Experimental brain research, 232(11), 3555-3567. doi:10.1007/s00221-014-4036-4

Vaquero, L., Hartmann, K., Ripollés, P., Rojo, N., Sierpowska, J., François, ... \& Münte, T. F. (2016). Structural neuroplasticity in expert pianists depends on the age of musical training onset. Neuroimage, 126, 106-119. doi:10.1016/j.neuroimage.2015.11.008

Welch, G. F., Howard, D. M., Himonides, E., \& Brereton, J. (2005). Real-time feedback in the singing studio: an innovatory action-research project using new voice technology. Music Education Research, 7(2), 225-249. doi:10.1080/14613800500169779 
Wilson, P. H., Lee, K., Callaghan, J., \& Thorpe, C. W. (2008). Learning to sing in tune: does realtime visual feedback help? Journal of Interdisciplinary Music Studies, 2, 157-172. 